\title{
Functional outcomes of platelet rich plasma injections in plantar fascitis
}

\author{
Niranjanan Raghavn Muralidharagopalan ${ }^{1 *}$, Dinesh Loganathan ${ }^{2}$, \\ Krithika Meenakshi Janakiram Iyer ${ }^{3}$, Kavin Kumar Boopathikumar ${ }^{4}$
}

\author{
Department Of Orthopaedics, ${ }^{1}$ Saveetha Medical College and Hospital, Saveetha Nagar, Thandalam, ${ }^{2}$ Chettinad \\ Medical College, Chennai, India \\ ${ }^{3}$ Nanganallur Bone and Joints Cliinic, Nanganallur, Chennai, India \\ ${ }^{4}$ Saveetha Medical College and Hospital, Saveetha Nagar, Thandalam, Chennai, India
}

Received: 20 April 2017

Revised: 01 May 2017

Accepted: 04 May 2017

\author{
*Correspondence: \\ Dr. Niranjanan Raghavn Muralidharagopalan, \\ E-mail: nir.rag@gmail.com
}

Copyright: () the author(s), publisher and licensee Medip Academy. This is an open-access article distributed under the terms of the Creative Commons Attribution Non-Commercial License, which permits unrestricted non-commercial use, distribution, and reproduction in any medium, provided the original work is properly cited.

\begin{abstract}
Background: The study was conducted to analyse the clinical outcomes following a single injection of platelet rich plasma, produced using autologous blood by standard double centrifugation technique in patients with plantar fasciitis who have not had relief using conservative methods.

Methods: Between June 2013 and June 2015, 26 consecutive patients (mean age 45.92) (11 males/15 females) who had symptoms for a minimum of 3 months and had failed conservative management options such as rest, antiinflammatories and physiotherapy were given a single injection of PRP in the medial calcaneal tubercle.

Results: The outcomes were measured using visual analogue scale and foot function index at pre injection, and at 1, 6 and 12 months post injection and results tabulated. All patients reported an improvement in VAS and FFI scores consistently. One superficial infection was encountered.

Conclusions: Single injection of platelet rich plasma improves pain and function in the foot in patients suffering from plantar fasciitis when conservative management has failed. The synergism between various autologous growth factors may play a role in tendon healing.
\end{abstract}

Keywords: Plantar fasciitis, Platelet rich plasma, Heel pain

\section{INTRODUCTION}

The plantar fascia is a thick aponeurtotic fascial band, which originates from the medial tubercle of the calcaneus and forms the longitudinal arch of the foot. The primary function of the plantar fascia is to provide static and dynamic support to the arch of the foot. ${ }^{1}$

Plantar fasciitis is the most common cause of heel pain and is usually diagnosed with a typical history and tenderness at the medial calcaneal tubercle. ${ }^{2,3}$ Plantar fasciitis is currently considered to be due to a degenerative pathology rather than an inflammatory pathology. ${ }^{4-6}$

The various first line treatment options include rest, physiotherapy and anti-inflammatory drugs. ${ }^{7,8}$ Local injections such as corticosteroids and local anaesthetics are used as second line of treatment. ${ }^{9,10}$

Platelet rich plasma (PRP) has been founded to increase the content of cytokines and promotes matrix synthesis and helps in tendon healing. ${ }^{11}$ 
Hence this study was conducted to study the effects of PRP on plantar fasciitis.

\section{Aim}

To study the clinical outcomes following the injection of platelet rich plasma in cases of plantar fasciitis

\section{METHODS}

26 consecutive patients who received platelet rich plasma in our institute for plantar fasciitis between June 2013 and June 2015 were included in the study. All 26 patients had failed conservative management such as rest analgesics and physiotherapy. All patients who had had a steroid injection before or had any concomitant pathology around the heel were excluded from the study.

Double centrifugation technique was used to concentrate the platelets from autologous blood. $25 \mathrm{ml}$ of venous blood was first centrifuged for 15 minutes at $1800 \mathrm{rpm}$ which separated the RBCs and then subsequently at 3500 rpm for 10 minutes. The PRP generated by this method was $\sim 3 \mathrm{ml}$.

The injection was given in the OR using sterile aseptic precautions. Patients were positioned prone and maximally tender points were identified. Skin puncture point was infiltrated with $2 \%$ lignocaine. Then the injection was made with the ankle neutral.

Post injection patients were not given any antiinflammatory or analgesic medications for a period of 2 weeks following the procedure. Ice pack application was done if needed.

Patients were analyzed using Visual analogue scale and Foot function index using standard questionnaires at pre injection and at 1,6 and 12 months post injection. ${ }^{12,13}$
The results were analyzed using Microsoft Excel for Mac 2013, Microsoft Inc USA.

\section{RESULTS}

Twenty-six patients were followed up for a minimum of one year.

Twenty-six patients, 11 males (42\%) and 15 females $(58 \%)$ were taken up for platelet rich plasma injection. Left side was involved in $53 \%(\mathrm{n}=14)$ of cases and the right side was involved in $47 \%$. Mean age group was 45.92 (range: $31-60$ years).

4 patients had type II diabetes mellitus, 3 patients had hypertension, 2 patients had hypothyroidism, 1 patient suffered from coronary artery disease, 1 patient had bronchial asthma and 1 patient had type II diabetes with hypertension.

The Visual analogue scores were tabulated pre-injection and at 1, 6 and 12 months post-injection. The visual analogue scale showed a decrease from $6 \pm 1$ at the pre injection levels to $1.42 \pm 1.08$ at one year follow-up.

The Foot function index scores were tabulated preinjection and at 1, 6 and 12 months post-injection. The

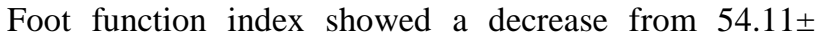
11.97 at the pre injection levels to $7.30 \pm 6.75$ at one year follow-up.

\section{Complications}

One patient developed superficial infection. She was treated with antibiotics and settled completely. There were no other complications.

All patients had a reduction in VAS and FFI scores, which indicated good improvement.

Table 1: Visual analogue scale.

\begin{tabular}{|llll|}
\hline & Mean \pm SD & Minimum & Maximum \\
\hline Pre injection & $6 \pm 1$ & 4 & 8 \\
\hline 1 month post injection & $3.96 \pm 1.01$ & 2 & 6 \\
\hline 6 months post injection & $2.07 \pm 0.82$ & 1 & 4 \\
\hline 12 months post injection & $1.42 \pm 1.08$ & 0 & 3 \\
\hline
\end{tabular}

Table 2: Foot function index.

\begin{tabular}{|llll|}
\hline & Mean \pm SD & Minimum & Maximum \\
\hline Pre injection & $54.11 \pm 11.97$ & 36 & 75 \\
\hline 1 month post injection & $35.57 \pm 11.34$ & 11 & 61 \\
\hline 6 months post injection & $13.96 \pm 7.98$ & 4 & 34 \\
\hline 12 months post injection & $7.30 \pm 6.75$ & 0 & 30 \\
\hline
\end{tabular}




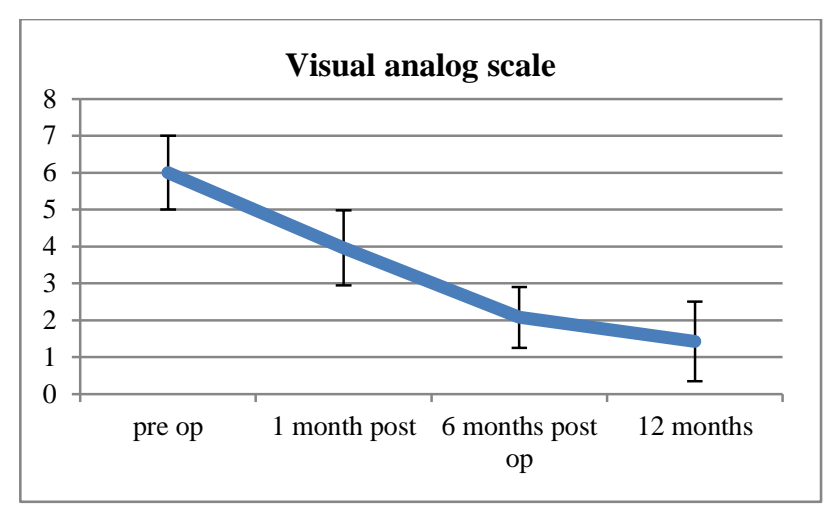

Figure 1: VAS improvement in scores over time. The graph shows a significant downward trend between pre injection and final follow up levels.

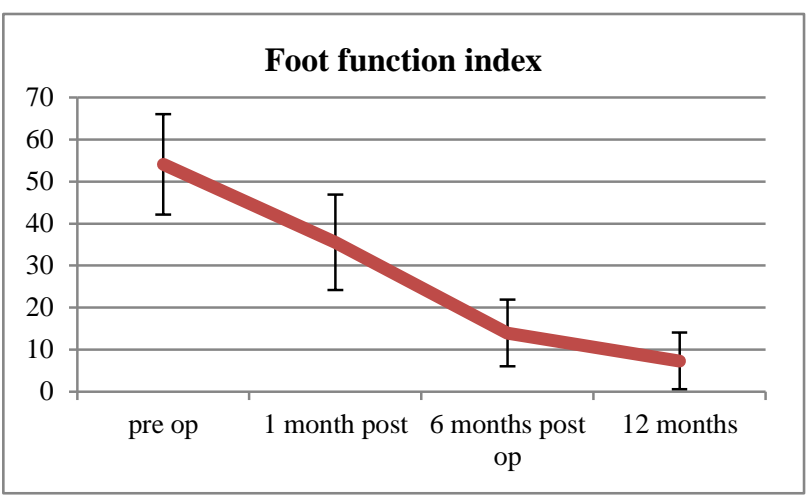

Figure 2: FFI Improvement in scores over time.

The graph shows a significant downward trend between pre injection and final follow up levels.

\section{DISCUSSION}

Plantar fasciitis is a chronic condition, which often does not get relieved with conservative measures such as analgesics, physiotherapy and rest.

Corticosteroid injections may give relief but only for a short duration. ${ }^{14,15}$ Also with the current concept moving more towards a degenerative aetiology anti-inflammatory effects of corticosteroids do not appear to be the best answer. $^{4-6}$

PRP has many autologous growth factors, which are helpful in the regeneration and growth of damaged ligaments and tendons. ${ }^{16,17}$

This also explains the persistent benefits of a single PRP injection, which lasts at least unto 1 year.

In the study of Aksahin et al the mean age was 46.36 years and the VAS scores at pre injection and 6 months post injection were $7.33[6-9]$ and 3.93 [0-8], which compared well with our study. ${ }^{18}$

In the study of Martinelli et al, 14 patients were studied. ${ }^{19}$ The mean age was 49.2 years and the VAS scores decreased significantly from $7.1 \pm 1.1$ before treatment to $1.9 \pm 1.5$ at the last follow-up, which was similar to our study.

Thus platelet rich plasma injection provides relief of pain and good functional outcome in treating plantar fasciitis.

\section{CONCLUSION}

Single injection of platelet rich plasma improves pain and function in the foot in patients suffering from plantar fasciitis when conservative management has failed. The synergism between various autologous growth factors may play a role in tendon healing.

Funding: No funding sources

Conflict of interest: None declared

Ethical approval: The study was approved by the institutional ethics committee

\section{REFERENCES}

1. Khan KM, Cook JL, Taunton JE, Bonar F. Over use tendinosis, not tendonitis; a new paradigm for a difficult clinical problem (part 1). Phys Sports Med 2000;28:172-5.

2. Pfeffer G, Bacchetti P, Deland J, Lewis A, Anderson R, Davis W, et al. Comparison of custom and prefabricated orthoses in the initial treatment of proximal plantar fasciitis. Foot Ankle Int. 1999;20:214-21.

3. Cole C, Seto C, Gazewood J. Plantar fasciitis: evidence-based review of diagnosis and therapy. Am Fam Physician. 2005;72:2237-42.

4. Jarde O, Diebold P, Havet E, Boulu G, Vernois J. Degenerative lesions of the plantar fascia: surgical treatment by fasciectomy and excision of the heel spur - a report on 38 cases. Acta Orthop Belg. 2003;69:267-74.

5. Leach RE, Seavey MS, Salter DK. Results of surgery in athletes with plantar fasciitis. Foot Ankle. 1986;7:156-61.

6. Lemont H, Ammirati KM, Usen N. Plantar fasciitis: a degenerative process (fasciosis) without inflammation. J Am Podiatr Med Assoc. 2003;93:234-7.

7. Lynch DM, Goforth WP, Martin JE, Odom RD, Preece CK, Kotter MW. Conservative treatment of plantar fasciitis: a prospective study. J Am Podiatr Med Assoc. 1998;88(8):375-80.

8. Young CC, Rutherford DS, Niedfeldt MW. Treatment of plantar fasciitis. Am Fam Physician. 2001;63:467-74.

9. KÂter E, Çelikbao E, Akkaya S, Demirkan F, Kiliç BA. Comparison of injection modalities in the treatment of plantar heel pain: a randomized controlled trial. J Am Podiatr Med Assoc. 2006;96:293-6.

10. Fadale PD, Wiggins ME Corticosteroid injections: their use and abuse. J Am Acad Orthop Surg. 1994;2:133-40. 
11. Marx RE. Platelet-rich plasma: evidence to support its use. J Oral Maxillofac Surg. 2004;62:489-96.

12. Carlsson AM. Assessment of chronic pain. I. Aspects of the reliability and validity of the visual analogue scale. Pain. 1983;16(1):87-101.

13. Budiman-Mak E, Conrad KJ, Roach KE. The Foot Function Index: a measure of foot pain and disability. J Clin Epidemiol. 1991;44(6):561-70.

14. Tatli YZ, Kapasi S. The real risks of steroid injection for plantar fasciitis, with a review of conservative therapies. Curr Rev Musculoskelet Med. 2009;2(1):3-9.

15. Ang TW. The effectiveness of corticosteroid injection in the treatment of plantar fasciitis. Singapore Med J. 2015;56(8):423-32.

16. Barrett S, Erredge S. Growth factors for chronic plantar fasciitis? Podiatry Today. 2004;17:37-42.

17. Peerbooms JC, Sluimer J, Bruijn D, Gosens T. Positive effect of an autologous platelet concentrate in lateral epicondylitis in a double-blind randomized controlled trial: platelet-rich plasma versus corticosteroid injection with a 1-year follow-up. Am J Sports Med. 2010;38:255-62.

18. Akşahin E, Doğruyol D, Yüksel HY, Hapa O, Doğan $\mathrm{O}$, Celebi L, et al. The comparison of the effect of corticosteroids and platelet-rich plasma (PRP) for the treatment of plantar fasciitis. Arch Orthop Trauma Surg. 2012;132(6):781-5.

19. Martinelli N, Marinozzi A. Platelet-rich plasma injections for chronic plantar fasciitis. Int Orthop. (SICOT). 2013;37:839-42.

Cite this article as: Muralidharagopalan NR, Loganathan D, Iyer KMJ, Boopathikumar KK. Functional outcomes of platelet rich plasma injections in plantar fascitis. Int J Res Orthop 2017;3:734-7. 